\title{
Cross-sectional association of volume, blood pressures, and aortic stiffness with left ventricular mass in incident hemodialysis patients: the Predictors of Arrhythmic and Cardiovascular Risk in End-Stage Renal Disease (PACE) study
}

Esther D. Kim', Stephen M. Sozio ${ }^{2,3}$, Michelle M. Estrella ${ }^{2,3}$, Bernard G. Jaar ${ }^{2,3,4}$, Tariq Shafi ${ }^{2,3}$, Lucy A. Meoni ${ }^{2,3,5}$, Wen Hong Linda Kao ${ }^{2,3,6}$, Joao A. C. Lima ${ }^{2}$ and Rulan S. Parekh ${ }^{1,2,6,7,8^{*}}$

\begin{abstract}
Background: Higher left ventricular mass (LV) strongly predicts cardiovascular mortality in hemodialysis patients. Although several parameters of preload and afterload have been associated with higher LV mass, whether these parameters independently predict LV mass, remains unclear.

Methods: This study examined a cohort of 391 adults with incident hemodialysis enrolled in the Predictors of Arrhythmic and Cardiovascular Risk in End Stage Renal Disease (PACE) study. The main exposures were systolic and diastolic blood pressure (BP), pulse pressure, arterial stiffness by pulse wave velocity (PWV), volume status estimated by pulmonary pressures using echocardiogram and intradialytic weight gain. The primary outcome was baseline left ventricular mass index (LVMI).
\end{abstract}

Results: Each systolic, diastolic blood, and pulse pressure measurement was significantly associated with LVMI by linear regression regardless of dialysis unit BP or non-dialysis day BP measurements. Adjusting for cardiovascular confounders, every $10 \mathrm{mmHg}$ increase in systolic or diastolic BP was significantly associated with higher LVMI (SBP $\beta=7.26,95 \% \mathrm{Cl}: 4.30,10.23$; DBP $\beta=10.05,95 \% \mathrm{Cl}: 5.06,15.04)$, and increased pulse pressure was also associated with higher LVMI ( $\beta=0.71,95 \% \mathrm{Cl}$ : 0.29, 1.13). Intradialytic weight gain was also associated with higher LVMI but attenuated effects after adjustment $(\beta=3.25,95 \% \mathrm{Cl}: 0.67,5.83)$. PWV and pulmonary pressures were not associated with $L V M I$ after multivariable adjustment ( $\beta=0.19,95 \% \mathrm{Cl}:-1.14,1.79$; and $\beta=0.10,95 \% \mathrm{Cl}:-0.51,0.70$, respectively). Simultaneously adjusting for all main exposures demonstrated that higher BP was independently associated with higher LVMI (SBP $\beta=5.64,95 \% \mathrm{Cl}: 2.78,8.49 ; \mathrm{DBP} \beta=7.29,95 \% \mathrm{Cl}: 2.26,12.31$, for every $10 \mathrm{mmHg}$ increase in BP).

Conclusions: Among a younger and incident hemodialysis population, higher systolic, diastolic, or pulse pressure, regardless of timing with dialysis, is most associated with higher LV mass. Future studies should consider the use of various BP measures in examining the impact of BP on LVM and cardiovascular disease. Findings from such studies could suggest that high BP should be more aggressively treated to promote LVH regression in incident hemodialysis patients.

\footnotetext{
* Correspondence: rulan.parekh@sickkids.ca

'Division of Epidemiology, Dalla Lana School of Public Health, University of

Toronto, Toronto, Ontario, Canada

2Department of Medicine, School of Medicine, Johns Hopkins University,

Baltimore, Maryland, USA

Full list of author information is available at the end of the article
}

\section{Biomed Central}

(c) 2015 Kim et al. Open Access This article is distributed under the terms of the Creative Commons Attribution 4.0 International License (http://creativecommons.org/licenses/by/4.0/), which permits unrestricted use, distribution, and reproduction in any medium, provided you give appropriate credit to the original author(s) and the source, provide a link to the Creative Commons license, and indicate if changes were made. The Creative Commons Public Domain Dedication waiver (http://creativecommons.org/publicdomain/zero/1.0/) applies to the data made available in this article, unless otherwise stated. 


\section{Background}

Left ventricular hypertrophy (LVH) is the most frequently observed cardiac abnormality in end-stage renal disease (ESRD), and strongly predicts cardiovascular mortality in hemodialysis patients [1]. Despite past findings demonstrating associations between various cardiac parameters and LVH [2-4], independent, modifiable, and clinically relevant predictors of left ventricular mass (LVM) in ESRD patients remain difficult to define. Clinical trials have shown that treatment of anemia and baseline factors such as cardiac troponin T, C-reactive protein, IL-6, and serum albumin are not associated with LV progression over follow-up [5]. Furthermore, the relative importance of various modifiable risk factors in predicting LVH in incident dialysis patients (i.e. patients transitioning from chronic kidney disease [CKD] to ESRD), in particular, remains unclear, as the majority of studies have included prevalent dialysis patients, predominantly Europeans, with potential survivor and selection bias as well as small sample sizes limiting analyses. African-Americans have also not been as extensively studied despite the high risk of hypertension and LVH prior to ESRD in this population.

Hypertension is a major risk factor for $\mathrm{LVH}$, and the role of blood pressure (BP) in the progression of LVM and mortality of patients receiving hemodialysis has been well documented [6]. The timing of BP measurements, however, has been controversial in dialysis patients, as BP levels are highly variable and BP measurements at a single time point may not be a reliable estimate of the arterial pressure load [7, 8].

Other factors associated with LVH are arterial stiffness and volume overload. Aortic pulse wave velocity (PWV) predicts cardiovascular disease and mortality in patients with hypertension and ESRD [9-11], and increased arterial stiffness measured by PWV is also associated with an increase in LVH or LVM in crosssectional analyses of prevalent dialysis patients $[2,12]$. Volume overload is closely associated with hypertension due to the positive sodium balance, which leads to hypertension and increased extracellular volume [13]. Studies have suggested that controlling for circulatory volume can decrease $\mathrm{BP}$, contribute to $\mathrm{LVH}$ regression, and improve survival $[13,14]$.

These parameters of arterial compliance and ventricular volume $[2,15]$, pre- and post-hemodialysis systolic blood pressure (SBP) [3, 4], and intradialytic weight gain (IDWG) $[13,14]$ have been implicated with higher left ventricular mass index (LVMI). However, these associations have not been consistently associated in studies likely due to the complicated interrelationship among these factors. Furthermore, there are only a few studies that incorporate all these vascular and volume measures and determine the independent associations of vascular, arterial, and volume measures with
LVM, as most studies with smaller sample sizes demonstrate that a single factor is associated with LVMI. However, clinically, it is important to understand which factor should be of most importance. This study therefore examines the relevant importance and the independent roles of blood pressure, arterial stiffness, and volume in predicting LVH using modifiable risk factors that are clinically relevant and available in a large prospective cohort of incident hemodialysis participants.

\section{Methods}

\section{Study participants}

We conducted cross-sectional analyses using data from participants enrolled in the Predictors of Arrhythmic and Cardiovascular Risk in End Stage Renal Disease (PACE) study, which enrolled 402 adult incident hemodialysis patients who completed a baseline cardiovascular study visit. The methods of the PACE study have been described in detail previously [16]. Briefly, PACE is a prospective cohort study in the greater Baltimore area, which enrolled participants from November 2008 to August 2012 from 27 outpatient dialysis units. Eligible participants were hemodialysis patients aged 18 years or older within six months of hemodialysis initiation, and able to provide informed consent. Participants were excluded if they were on home dialysis, had active cancer other than nonmelanoma skin cancer, had a pacemaker/automatic implantable cardiac defibrillator, had detectable atrial fibrillation at the time of echocardiogram, were pregnant, were nursing mothers or were non-English speaking patients. In this analysis, participants without LVMI measurements were further excluded.

The study protocol was approved by the institutional review board of the Johns Hopkins School of Medicine, MedStar Health Systems, and by the medical director of each dialysis unit. All participants provided informed written consent.

\section{Data collection}

Data were collected by self-report from standardized questionnaires and also during study visits at the Johns Hopkins Institute for Clinical and Translational Research (ICTR). All participants underwent a study visit with detailed cardiovascular evaluations by trained technologists or study staff including echocardiogram, PWV and BP assessments on non-dialysis days to ensure uniformity across the study population. Based on the studies of myocardial stunning during dialysis and at the end of dialysis treatments with many shifts in fluids and electrolytes, the study visit was set to a non-dialysis day in order to standardize the study visits for all dialysis patients and asses their cardiac status at a steady state. Data on all hemodialysis treatment parameters and available laboratory tests during outpatient dialysis therapy 
were also provided by Davita Clinical Research and MedStar Health Systems.

The echocardiograms (Toshiba Artida, Japan) were performed by three trained technologists at baseline, to determine left ventricular (LV) stroke volume, LV ejection fraction, and LVMI with 4 chamber views and standard calculations for LVM. The M-mode by the parasternal short axis view was used to estimate LVM, as the long axis view can result in the improper alignment and overestimate LV dimensions and mass [17]. All cardiovascular studies were centrally read. Very few participants at baseline had heart failure indicated by low ejection fraction (1\%). Additionally, we estimated pulmonary artery pressures based on measured velocity of the tricuspid regurgitant (TR) jet. Aortic PWV measures were performed supine on the non-fistula arm after at least $5 \mathrm{~min}$ of rest using the right carotid and right femoral arteries by four investigators, and the augmentation index was measured using radial tonometry using the Sphygmocor PVx System (AtCor Medical, West Ryde, Australia) device [18]. PWV measurements were performed in the morning under similar conditions in all participants and appropriate quality control measures were performed. Additionally, patients were fasting prior to the assessment [18].

The exposure variables of interest included measures of volume (pulmonary artery pressures and IDWG), and vascular and arterial measurements. Vascular measurements included SBP and diastolic blood pressure (DBP), mean arterial pressure (MAP), pulse pressure, PWV, and augmentation index. Four different measurements of SBP or DBP and pulse pressure at various time points were included. Predialysis BP measures taken at the dialysis units were examined as (1) a three-month average of the predialysis measurements taken using automated BP measurements prior to the study visit and (2) a single measurement closest to the study visit. Non-dialysis BP measures taken at the study vist on a non-dialysis day were examined as: (1) an average of three standardized BP measures conducted using the Omron HEM-907 BP measuring device (Omron Healthcare, Kyoto, Japan) in a seated and (2) an average of three BP measures collected using the same method in a supine position. Volume status was estimated by pulmonary artery pressure based on TR from the echocardiogram [19-21], as the presence of pulmonary hypertension occurs when TR jet velocity exceeds $3.4 \mathrm{~m} / \mathrm{s}$ [22]. Furthermore, echocardiographic parameters such as left atrial diameters, which may be reflective of the filling pressure and influenced by TR, have been associated with excess volume in hemodialysis (HD) patients [23]. Volume measurement also included IDWG, and this was analyzed as: 1) a three-month average of IDWG measurements taken at the dialysis units, and 2) a measurement closest to the study visit.
The primary endpoint was LVMI calculated by Devereux's formula [24], as recommended by the American Society of Echocardiography [17], using left ventricular internal diameter (LVID), interventricular septal thickness (IVST) and left ventricular posterior wall thickness (PWT):

$$
\begin{aligned}
\operatorname{LVMI}\left(\mathrm{g} / \mathrm{m}^{2}\right)= & \left(0.8\left(1.04\left[(\mathrm{IVST}+\mathrm{LVID}+\mathrm{PWT})^{3}-\mathrm{LVID}^{3}\right]\right)\right. \\
& +0.6 \mathrm{~g}) / \mathrm{BSA}
\end{aligned}
$$

LVH was defined as LVMI greater than $116 \mathrm{~g} / \mathrm{m}^{2}$ in males and LVMI greater than $104 \mathrm{~g} / \mathrm{m}^{2}$ in females.

Additional variables included self-reported age at enrollment, sex, and ethnicity. Body-mass index (BMI) and waist to hip ratio (WHR) were determined during the ICTR visits. Comorbidities were identified by detailed chart review of medical records and included history of hypertension, hypercholesterolemia, smoking status, diabetes mellitus, coronary artery disease (CAD), congestive heart failure (CHF) and peripheral vascular disease (PVD), dialysis frequency, cause of ESRD, time since first nephrology visit, antihypertensive medications, and the Charlson Comorbidity Index (CCI). Medications were determined at baseline by medical record review from dialysis electronic patient records and participant questionnaire during the study visit. Medications were also brought in for review by the study team. As a result, adherence to medication treatment was not specifically assessed. Antihypertensive medications were grouped into the following categories: (1) Renin-angiotensin-aldosterone system (RAAS) blockade (angiotensin-converting-enzyme inhibitor [ACEi] or angiotensin II receptor blocker [ARB]), (2) $\beta$-blocker or $\alpha$ - and $\beta$-blocker, (3) $\alpha$-blocker, vasodilator, or calcium channel blocker, (4) centrally acting agent, and (5) other. Dietary sodium intake was calculated from a 24-h diet recall at the baseline study visit. Dialysis unit hemoglobin data were included as a 3 month average prior to the study visit.

\section{Statistical analysis}

For categorical variables, frequencies and percentages with $95 \%$ confidence intervals $(\mathrm{CI})$ were used; for continuous variables, means with standard deviations $( \pm \mathrm{SD})$ were used for normally distributed data and median with interquartile range for skewed distributed data.

Multiple analyses were used to examine the association of BP, arterial stiffness, and volume with LVMI. To assess the associations of each vascular, arterial and volume measurement with LVMI, a multivariable linear regression for each vascular, arterial or volume measure was conducted. To assess the independent association of each main explanatory measure with LVMI, all vascular, arterial, and volume measures were simultaneously adjusted for in the final multivariable linear regression model. Each 
vascular measure was modeled in a separate multivariable model due to the high correlation between the vascular measurements. The multivariable models were constructed using the forward selection model building approach, and included age, sex, ethnicity, BMI, history of hypercholesterolemia, smoking, diabetes mellitus, coronary heart disease, congestive heart failure, cause of endstage renal disease, time since first nephrology visit, beta blocker and RAAS blockade use, number of antihypertensive medication, and dietary sodium intake. To ensure the consistency and the validity of the final model, all omitted variables were included back into the final model and this indicated that there were no significant changes in the main estimates. Correlation and variance inflation factor tests indicated a low degree of multicollinearity among all factors. Tests of non-linearity indicated that there were no significant non-linear effects. Patients with missing antihypertensive medication data (14\%) were grouped into a separate category. All other missing values for covariates were imputed using the multiple imputation by chained equations method [25]. The imputed variables with missing values were WHR (4.1\%), history of hypercholesterolemia (4.3\%), CCI (2.6\%), dietary sodium intake (5.9\%), and time since first nephrology visit (1.0\%).

Given the high proportion of African American patients in this study, we tested for an interaction between the exposure variables and ethnicity (African Americans vs. non-African Americans) in stratified models. We also stratified the results from the multivariable regression models using pre-identified groups of IDWG $(0-2 \mathrm{~kg}$, $2-3 \mathrm{~kg}$, and $>3 \mathrm{~kg}$ ), by $\beta$-blocker medication status [26], and by RAAS blockade use.

A two-tailed $P$ value of $<0.05$ was considered statistically significant. All analyses were performed using Stata/SE 12.0 (StataCorp, College Station, TX).

\section{Results}

Of the 402 participants who completed the study visits with an echocardiogram, 11 were excluded due to poor echocardiographic windows for measurement of LVMI. The final study cohort comprised of 391 patients. Table 1 summarizes the baseline participant, vascular, volume, and echocardiogram measurement characteristics. The study population has mean age at enrollment of 54.7 years, median BMI of $27.9 \mathrm{~kg} / \mathrm{m}^{2}$ and are predominantly male and African American. Cardiovascular risk factors are common: $57 \%$ with diabetes, $33 \%$ with CAD, $41 \%$ with CHF, $20 \%$ with PVD, and $62 \%$ has a history of smoking. The vast majority is on two to three antihypertensive medications with approximately $62 \%$ taking a beta-blocker and $39 \%$ on any RAAS blockade. A total of 306 patients (78 \%) have demonstrable LVH.

Figure 1 illustrates side-by-side scatterplots of LVMI, SBP, and DBP by type of BP measurement. In all four measurements of BP, SBP and DBP have graded linear associations with LVMI; however, DBP consistently shows a steeper slope with LVMI than SBP. These patterns are consistent regardless of timing of the BP measurement. Figure 2 illustrates the quartiles of non-dialysis seated BP measurements and trends of LVMI, stratified by ethnicity. The plots demonstrate a significant positive association between quartiles of SBP and DBP, and LVMI among African Americans. Among non-African Americans, only SBP is significantly associated with LVMI. Similar associations are depicted using other BP measurements (Additional file 1: Figure S1-S3).

The associations of vascular and volume measures with LVMI are examined and shown in Table 2. The unadjusted linear regression model demonstrates that, among vascular measures, BP, pulse pressure, and MAP are all associated with higher LVMI. In particular, all SBPs and DBPs, regardless of the timing of the measurements, are most significantly associated with LVMI. Among volume measures, only IDWG just prior to the study visit is significantly associated with higher LVMI. Adjusting for baseline covariates demonstrates that BP measures remains significantly associated with higher LVMI and the association with IDWG is attenuated. In particular, DBPs are the most important factors among BPs. Measures of arterial stiffness or pulmonary pressure, after multivariable adjustment, are not associated with LVMI.

Table 3 demonstrates the independent associations of non-dialysis seated BP, PWV, and volume measures with LVMI using a multivariable linear regression model that simultaneously adjusted for the main explanatory measures and other covariates. Among all BP, arterial, and volume measurements, SBP and DBP are independently associated with LVMI. Similar associations are demonstrated using other vascular measures (predialysis, pulse pressure, mean arterial pressure; Additional file 1: Table S1-S4). PWV and IDWG measures are not independently associated with LVMI.

Given the high proportion of African American patients, we first examined the interaction between ethnicity and the exposure variables, and then stratified the results from the adjusted model (Additional file 1: Table S5). The stratified analysis by ethnicity demonstrates that in African Americans, most BPs, pulse pressures, and MAP are significantly associated with LVMI, whereas in non-African Americans, few factors were associated with LVMI (Additional file 1: Table S5). There are no significant interactions between ethnicity and the explanatory variables.

We also postulated that the associations of BPs and LVMI might not be constant across participants with varying IDWG. In the stratified analyses (Additional file 1: Table S6), among those in the lowest IDWG group 
Table 1 Baseline characteristics of incident hemodialysis participants

\begin{tabular}{|c|c|c|}
\hline \multirow[t]{2}{*}{ Variables } & $\begin{array}{l}\text { Number of } \\
\text { patients }\end{array}$ & $\begin{array}{l}\mathrm{n}(\%) \text { or mean }( \pm \mathrm{SD}) \\
\text { or median }(\mathrm{IQR})\end{array}$ \\
\hline & \multicolumn{2}{|c|}{$(N=391)$} \\
\hline Age (years) & 391 & $54.7( \pm 13.2)$ \\
\hline Male & 391 & $229(58.6 \%)$ \\
\hline \multicolumn{3}{|l|}{ Ethnicity } \\
\hline African American & 391 & $284(72.6 \%)$ \\
\hline Non-African American & & $107(27.4 \%)$ \\
\hline Body mass index $\left(\mathrm{kg} / \mathrm{m}^{2}\right)$ & 391 & $27.9(23.8,33.2)$ \\
\hline Waist to hip ratio & 375 & $0.95( \pm 0.08)$ \\
\hline Ever smoker & 391 & $242(61.9 \%)$ \\
\hline Hypercholesterolemia & 374 & $225(57.5 \%)$ \\
\hline Hypertension & 391 & $390(99.7 \%)$ \\
\hline Diabetes mellitus & 391 & $224(57.3 \%)$ \\
\hline Coronary artery disease & 391 & $130(33.3 \%)$ \\
\hline Congestive heart failure & 391 & $160(40.9 \%)$ \\
\hline Peripheral vascular disease & 387 & 77 (19.9 \%) \\
\hline Dietary sodium intake (mg) & 368 & $\begin{array}{l}2219.1(1370.3 \\
\quad 3045.1)\end{array}$ \\
\hline $\begin{array}{l}\text { Hemoglobin (predialysis } 3 \text { month } \\
\text { average) }\end{array}$ & 361 & $10.5( \pm 1.2)$ \\
\hline Charlson comorbidity index & 381 & $5(3,6)$ \\
\hline \multicolumn{3}{|l|}{ Medications } \\
\hline ACEi or ARB & 336 & $154(46.6 \%)$ \\
\hline$\beta$-blocker or $a$ - and $\beta$-blocker & 336 & $242(61.9 \%)$ \\
\hline $\begin{array}{l}\text { a-blocker or vasoilator or calcium } \\
\text { channel blocker }\end{array}$ & 336 & $271(69.3 \%)$ \\
\hline Centrally acting agent & 336 & $47(12.0 \%)$ \\
\hline Diuretic & 336 & $80(20.5 \%)$ \\
\hline \multicolumn{3}{|l|}{$\begin{array}{l}\text { Total number of antihypertensive } \\
\text { medications }\end{array}$} \\
\hline 1 medication & 336 & $48(12.3 \%)$ \\
\hline 2 medications & & $109(27.9 \%)$ \\
\hline 3 medications & & $106(27.1 \%)$ \\
\hline$>4$ medications & & $73(18.7 \%)$ \\
\hline \multicolumn{3}{|l|}{ Cause of end-stage renal disease } \\
\hline Diabetes & 391 & $136(34.8 \%)$ \\
\hline Hypertension & & $100(25.6 \%)$ \\
\hline Glomerulonephritis & & $54(13.8 \%)$ \\
\hline Other & & $62(15.9 \%)$ \\
\hline Unknown & & $39(10.0 \%)$ \\
\hline \multicolumn{3}{|l|}{ Time since first nephrology visit } \\
\hline 0 months & 387 & $83(21.2 \%)$ \\
\hline$<3$ months & & $53(13.6 \%)$ \\
\hline 3 months - 12 months & & $68(17.4 \%)$ \\
\hline$>12$ months & & $183(46.8 \%)$ \\
\hline
\end{tabular}

Table 1 Baseline characteristics of incident hemodialysis participants (Continued)

\begin{tabular}{|c|c|c|}
\hline Three times a week dialysis & 386 & $386(98.7 \%)$ \\
\hline Three to four hour dialysis session & 389 & $344(88.0 \%)$ \\
\hline \multicolumn{3}{|l|}{ Hemodialysis access } \\
\hline Arteriovenous fistula & 389 & $120(30.7 \%)$ \\
\hline Other & & $271(69.3 \%)$ \\
\hline Polyflux dialysis membrane ${ }^{a}$ & 391 & 300 (76.7 \%) \\
\hline \multicolumn{3}{|l|}{ Vascular Measurements } \\
\hline \multicolumn{3}{|l|}{ Blood pressure (mmHg) } \\
\hline \multicolumn{3}{|l|}{ Predialysis (3 month average) } \\
\hline Systolic & 383 & $153.0( \pm 16.8)$ \\
\hline Diastolic & & $84.0( \pm 11.3)$ \\
\hline \multicolumn{3}{|l|}{ Predialysis (Prior to study visit) } \\
\hline Systolic & 380 & $154.9( \pm 26.0)$ \\
\hline Diastolic & & $84.2( \pm 15.8)$ \\
\hline \multicolumn{3}{|l|}{ Non-dialysis (Seated) } \\
\hline Systolic & 387 & $136.6( \pm 25.2)$ \\
\hline Diastolic & & $74.5( \pm 14.9)$ \\
\hline \multicolumn{3}{|l|}{ Non-dialysis (Supine) } \\
\hline Systolic & 367 & $148.0( \pm 25.4)$ \\
\hline Diastolic & & $79.1( \pm 15.2)$ \\
\hline $\begin{array}{l}\text { Mean arterial pressure } \\
\text { (Non-dialysis) }\end{array}$ & 370 & $103.0( \pm 19.7)$ \\
\hline \multicolumn{3}{|l|}{ Pulse pressure } \\
\hline Predialysis (3 months average) & 383 & $69.0( \pm 13.1)$ \\
\hline Predialysis (Prior to study visit) & 380 & $69.7( \pm 20.1)$ \\
\hline Non-dialysis (Seated) & 387 & $62.1( \pm 17.9)$ \\
\hline Non-dialysis (Supine) & 367 & $68.8( \pm 18.3)$ \\
\hline
\end{tabular}

Arterial Stiffness Measurements

Pulse wave velocity $(\mathrm{m} / \mathrm{s}) \quad 337$

$11.2( \pm 3.9)$

Central augmentation index (\%) $\quad 365$

$25.4(19.0,34.0)$

Volume Measurements

Tricuspid regurgitation 21

(Non-dialysis)

Intradialytic weight gain (3 month 383 average) $(\mathrm{kg})$

Intradialytic weight gain (Prior to study visit) (kg)

$380 \quad 2.6( \pm 1.9)$

Echocardiogram Measurements

$\begin{array}{lll}\text { Left ventricular ejection fraction } & 391 & 65.3( \pm 12.0) \\ \text { Left ventricular mass }(\mathrm{g}) & 391 & 286.5( \pm 98.7) \\ \text { Left ventricular mass index }\left(\mathrm{g} / \mathrm{m}^{2}\right) & 391 & 150.0( \pm 49.0) \\ \text { Left ventricular hypertrophy }^{\mathrm{b}} & 391 & 306(78.3 \%)\end{array}$

$A R B$ angiotensin II receptor blocker, $A C E I$ angiotensin-converting-enzyme inhibitor

apolyflux, Gambro dialyzer with a polymer blend of polyarylethersulfone, polyvinylpyrrolidone, and polyamide dialysis membrane

${ }^{b}$ LVMI $\geq 116 \mathrm{~g} / \mathrm{m}^{2}$ in males, $\geq 104 \mathrm{~g} / \mathrm{m}^{2}$ in females 

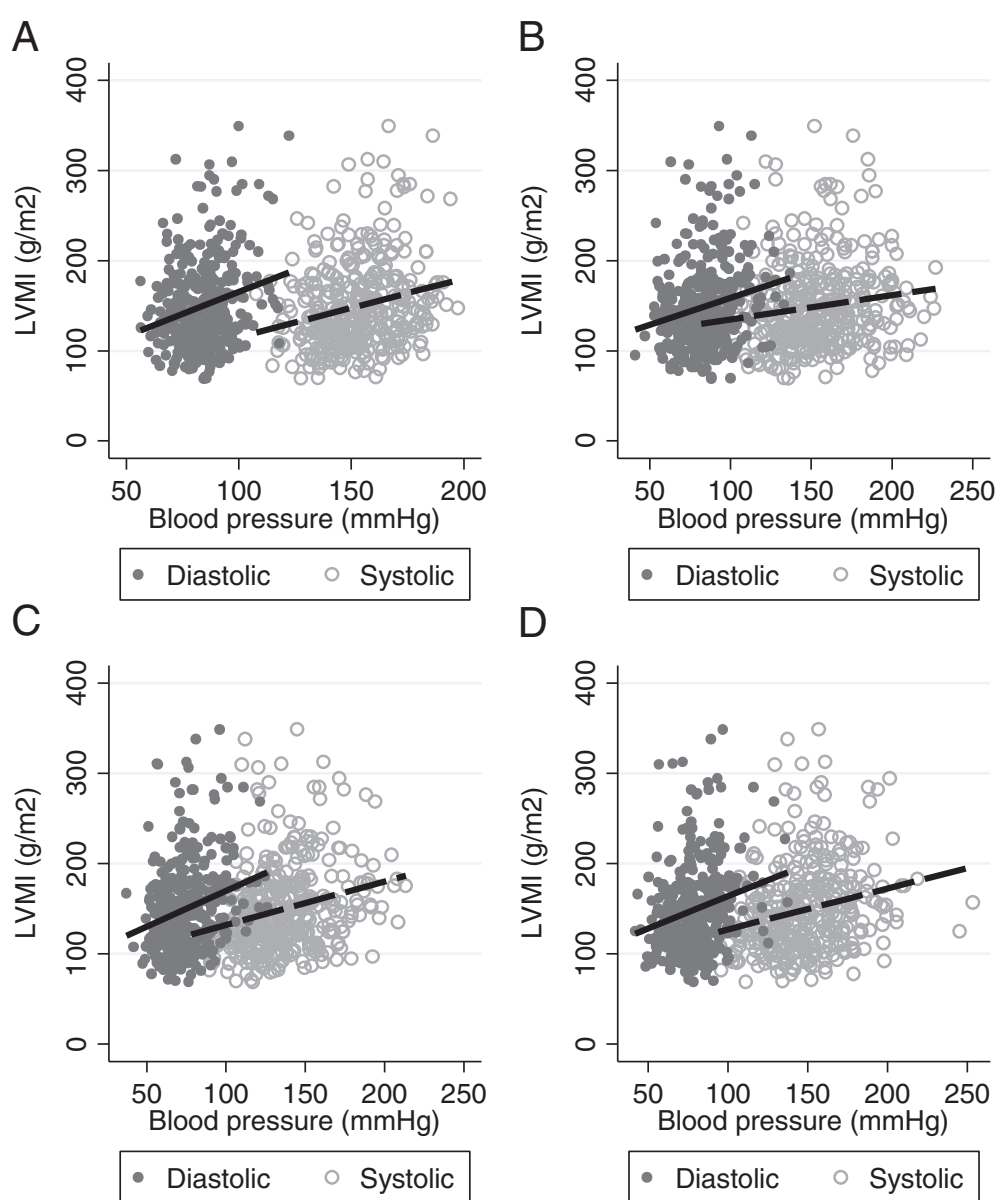

$\mathrm{D}$

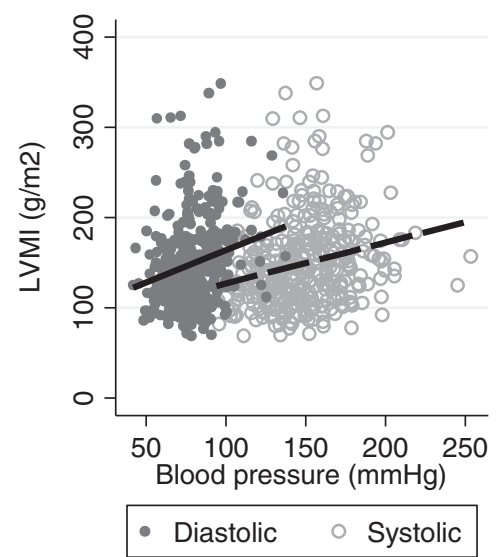

Fig. 1 Scatterplots of left ventricular mass index (LVMI) and systolic and diastolic blood pressure at various time points as a three-month average of the predialysis measurement prior to the study visit; $\mathbf{b}$ predialysis measurement prior to the study visit; $\mathbf{c}$ seated non-dialysis BP measurement and $\mathbf{d}$ supine non-dialysis measurement

$(<2 \mathrm{~kg})$, most BP measurements and pulse pressures are significantly associated with LVMI. In the intermediate IDWG group (2-3 kg), most BP measures are significantly associated with LVMI. In the highest IDWG group (>3 kg), no measures are associated with LVMI; however, the sample size is extremely small.

Based on recent trial findings that demonstrate a difference in cardiovascular morbidity between $\beta$-blocker- and ACEi-based therapy [26], we also stratified by $\beta$-blocker medication status (Additional file 1: Table S7) and RAAS blockade use (Additional file 1: Table S8). Among patients who received no $\beta$-blockers, some BP measures and IDWG prior to study visit are significantly associated with higher LVMI. Among patients who received $\beta$-blocker medications, most vascular measures are associated with higher LVMI. There are no significant differences in the mean BPs between patients who received $\beta$-blockers and did not receive $\beta$-blockers. In patients who received RAAS blockade, most vascular measures are significantly associated with higher LVMI. In patients who did not receive
RAAS blockade, few measures are associated with higher LVMI. There are also no differences in the mean BPs between patients who did and did not receive RAAS blockade.

\section{Discussion}

Among an ethnically diverse cohort incident to hemodialysis, measures of afterload are consistently and independently associated with higher LVMI in a linear graded response even after controlling for other cardiovascular disease risk factors and antihypertensive medications. Arterial stiffness and pulmonary pressure are not associated with LVMI. This suggests that, of all vascular, arterial, and volume measures, afterload reduction as measured by BP is an important factor associated with higher LVMI in those starting dialysis, and thus significant in managing cardiovascular risk for those patients during the early initiation of hemodialysis.

Our study extends findings from the longitudinal Chronic Renal Insufficiency Cohort (CRIC) study, which 

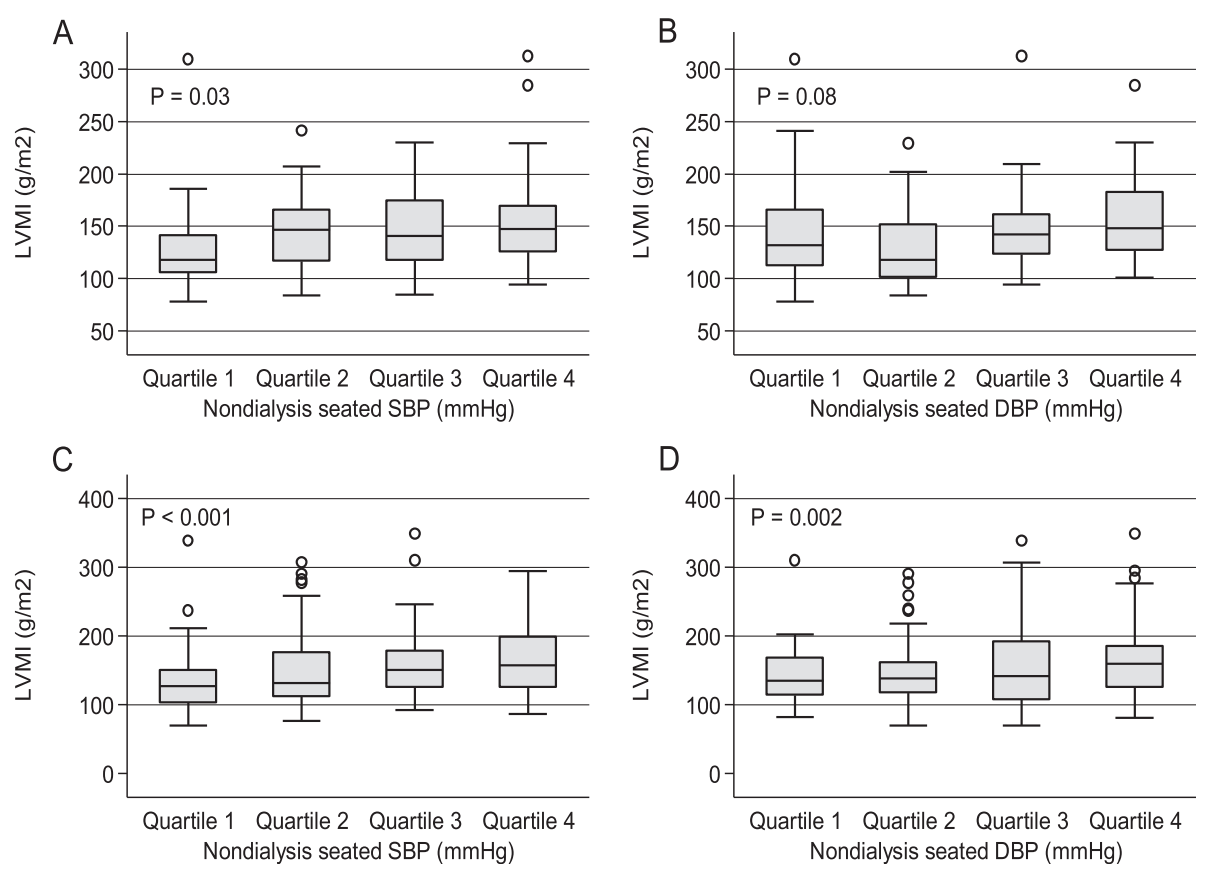

Fig. 2 Boxplot of left ventricular mass index (LVMI) and quartiles of systolic or diastolic blood pressures measured as a seated non-dialysis BP measurement stratified by ethnicity $\mathbf{a}$ and $\mathbf{b}$ non-African Americans and $\mathbf{c}$ and $\mathbf{d}$ African American participants

examines changes in LV structure and function in patients transitioning from CKD to ESRD but could not examine the role of clinical factors associated with LV disease [27]. We are able to demonstrate, in a similar but larger population of incident HD patients, significant associations of modifiable risk factors, in particular, blood and pulse pressures, with LVM. Our findings are also consistent in a subgroup of patients without a prior history of CHF (Additional file 1: Table S9). We report fewer patients with LVH and mostly with preserved cardiac function, which may be reflective of a younger and more ethnically diverse study population. Our findings also confirm and extend findings from the Erythropoietin Normalization trial that showed a significant association between higher SBP and LVM [5], as we are able to demonstrate consistently higher LVMI with either SBPs or DBPs at various time points and controlling for measures of volume status and arterial stiffness and not just SBP. Furthermore, no significant associations between hemoglobin and LVMI were found in our analysis (Additional file 1: Table S10). Recent reviews have suggested using non-dialysis BPs for management, however, it is complicated to obtain morning BPs unless patients are capable of home monitoring $[28,29]$. A 24-h ambulatory $\mathrm{BP}$ monitoring would provide the best $\mathrm{BP}$ measurements [30], but this is costly and impractical for all dialysis patients [31]. Although both systolic and diastolic BPs are associated with higher LVMI in this study, DBP consistently shows a stronger association with
LVMI in comparison to SBP. This finding deviates from earlier studies where higher predialysis SBP is the main arterial hemodynamic factor associated with $\mathrm{LVH}$ progression [32]. The stronger association of DBP with LVMI in our study could be attributed to the predominantly African American study population or due to a severely affected diseased population. Comparison of SBP, DBP and pulse pressure with mortality on dialysis patients demonstrated that DBP significantly attenuates the association of SPB and pulse pressure on mortality and thus is an important factor leading to mortality [33]. The association between higher DBP and higher LV mass may be indicative of lower LV diastolic function in our cohort, which is often a consequence of LVH. A meta-analysis of trials has also demonstrated that the reduction of diastolic BP in particular is important in the regression of LVH [34]. Nonetheless, the direction of effect is consistent for either systolic or diastolic pressures.

Despite the findings of previous studies, evidence to guide BP management in dialysis patients still remains sparse [30]. The guidelines from the Kidney Disease Outcomes Quality Initiative and the Joint National Committee suggest that the goal BP should be less than $140 / 90 \mathrm{mmHg}$ $[35,36]$, however, the Kidney Disease: Improving Global Outcomes states that these recommendations are largely based on expert opinion and weak evidence, and does not provide a recommendation regarding BP management in dialysis patients [30]. Our results show that approximately $79 \%$ of those receiving dialysis with a predialysis BP 
Table 2 Association of vascular, arterial, and volume measures with LVMI by linear regression among incident hemodialysis participants

\begin{tabular}{|c|c|c|c|c|c|}
\hline \multirow[t]{2}{*}{ Variables } & \multirow[t]{2}{*}{$\mathrm{n}$} & \multicolumn{2}{|l|}{ Univariable } & \multicolumn{2}{|l|}{ Multivariable $^{a}$} \\
\hline & & $\beta(95 \% \mathrm{Cl})$ & $P$ & $\beta(95 \% \mathrm{Cl})$ & $P$ \\
\hline \multicolumn{6}{|l|}{ Vascular measurements } \\
\hline \multicolumn{6}{|l|}{ Blood pressure (per 10 mmHg) } \\
\hline Predialysis (3 month average) & 383 & & & & \\
\hline Systolic & & $6.51(3.69,9.33)$ & $<0.001$ & $7.26(4.30,10.23)$ & $<0.001$ \\
\hline Diastolic & & $9.71(5.52,13.90)$ & $<0.001$ & $10.05(5.06,15.04)$ & $<0.001$ \\
\hline Predialysis (Prior to study visit) & 380 & & & & \\
\hline Systolic & & $2.69(0.83,4.56)$ & 0.005 & $2.46(0.57,4.35)$ & 0.01 \\
\hline Diastolic & & $5.95(2.92,8.99)$ & $<0.001$ & $3.93(0.67,7.20)$ & 0.02 \\
\hline Non-dialysis (Seated) & 387 & & & & \\
\hline Systolic & & $4.83(2.94,6.72)$ & $<0.001$ & $5.00(3.11,6.89)$ & $<0.001$ \\
\hline Diastolic & & $7.87(4.68,11.06)$ & $<0.001$ & $7.28(3.79,10.77)$ & $<0.001$ \\
\hline Non-dialysis (Supine) & 367 & & & & \\
\hline Systolic & & $4.54(2.62,6.46)$ & $<0.001$ & $4.72(2.72,6.72)$ & $<0.001$ \\
\hline Diastolic & & $7.13(3.91,10.35)$ & $<0.001$ & $6.25(2.64,9.85)$ & 0.001 \\
\hline Mean arterial pressure (Non-dialysis) & 370 & $0.57(0.33,0.82)$ & $<0.001$ & $0.56(0.29,0.82)$ & $<0.001$ \\
\hline \multicolumn{6}{|l|}{ Pulse pressure } \\
\hline Predialysis (3 months average) & 383 & $0.35(-0.02,0.72)$ & 0.07 & $0.71(0.29,1.13)$ & 0.001 \\
\hline Predialysis (Prior to study visit) & 380 & $0.12(-0.13,0.37)$ & 0.34 & $0.26(-0.01,0.53)$ & 0.05 \\
\hline Non-dialysis (Seated) & 387 & $0.41(0.13,0.68)$ & 0.003 & $0.65(0.36,0.94)$ & $<0.001$ \\
\hline Non-dialysis (Supine) & 367 & $0.38(0.11,0.65)$ & 0.006 & $0.60(0.30,0.89)$ & $<0.001$ \\
\hline \multicolumn{6}{|l|}{ Arterial stiffness measurements } \\
\hline Pulse wave velocity (m/s) & 337 & $-0.42(-1.78,0.94)$ & 0.55 & $0.19(-1.41,1.79)$ & 0.82 \\
\hline Central augmentation index & 365 & $-0.12(-0.48,0.25)$ & 0.53 & $-0.03(-0.41,0.36)$ & 0.88 \\
\hline \multicolumn{6}{|l|}{ Volume measurements } \\
\hline Tricuspid regurgitation (Non-dialysis) & 211 & $0.32(-0.29,0.92)$ & 0.30 & $0.10(-0.51,0.70)$ & 0.76 \\
\hline Intradialytic weight gain (3 month average) & 383 & $3.75(-0.51,8.01)$ & 0.09 & $4.36(-0.26,8.98)$ & 0.06 \\
\hline Intradialytic weight gain (Prior to study visit) & 380 & $3.77(1.17,6.37)$ & 0.005 & $3.25(0.67,5.83)$ & 0.01 \\
\hline
\end{tabular}

${ }^{a}$ Adjusted model included age, sex, ethnicity, body mass index, history of hypercholesterolemia, smoking, diabetes mellitus, coronary heart disease, congestive heart failure, cause of end-stage renal disease, time since first nephrology visit, beta blocker use, RAAS blockade use, number of antihypertensive medication, and dietary sodium intake

greater than 140/90 mmHg had higher LVMI. Furthermore, our study reflects the proportion of patients at dialysis initiation as they transition from advanced CKD to hemodialysis, and this provides a window to potentially mitigate cardiovascular risk on dialysis by aggressively treating high $\mathrm{BP}$ at early stages of dialysis. We are also able to demonstrate, in our large incident cohort, that BP is associated with higher LVMI regardless of timing. Clinicians could use predialysis measures for adjustment in medical management.

Other controversies regarding the BP management in dialysis patients include the effect of BP-lowering medications, such as RAAS inhibitors, $\beta$-blockers, and calcium channel blockers, on the reduction of LVMI in hypertensive patients [37, 38]. The Hypertension in
Hemodialysis Patients Treated with Atenolol or Lisinopril (HDPAL) trial demonstrates the beneficial effect of $\beta$ blocker to RAAS blockade in preventing cardiovascular morbidity [26]. Our findings suggest that those on either a $\beta$-blocker or RAAS blockade may have a protective role but this needs to be confirmed by larger randomized controlled trials with specifically targeted BP levels.

Contrary to past studies, we do not find that vascular stiffness was significantly associated with LVMI [12]. Arterial stiffness may be a factor associated with LV disease over prolonged dialysis, as vascular calcification can worsens and contribute to increased arterial stiffness $[12,39]$. Our study also includes incident and significantly younger HD patients, whereas most studies include prevalent and older patients over age 65 years, 
Table 3 Independent associations of non-dialysis seated blood pressure, arterial, and volume measures with LVMI by linear regression among incident hemodialysis participants

\begin{tabular}{|c|c|c|c|c|}
\hline \multirow[t]{3}{*}{ Variables } & \multicolumn{2}{|l|}{ Model $1^{a}$} & \multicolumn{2}{|l|}{ Model $2^{b}$} \\
\hline & \multicolumn{2}{|l|}{$(n=177)$} & \multicolumn{2}{|l|}{$(n=177)$} \\
\hline & $\beta(95 \% \mathrm{Cl})$ & P & $\beta(95 \% \mathrm{Cl})$ & $P$ \\
\hline \multicolumn{5}{|l|}{ Vascular measurements } \\
\hline \multicolumn{5}{|l|}{ Blood pressure (per 10 mmHg) } \\
\hline Non-dialysis seated systolic & $5.64(2.78,8.49)$ & $<0.001$ & - & - \\
\hline Non-dialysis seated diastolic & - & - & $7.29(2.26,12.31)$ & 0.005 \\
\hline \multicolumn{5}{|l|}{ Arterial stiffness measurement } \\
\hline Pulse wave velocity $(\mathrm{m} / \mathrm{s})$ & $-0.05(-2.44,2.34)$ & 0.97 & $0.41(-2.01,2.84)$ & 0.74 \\
\hline Central augmentation index & $-0.36(-0.88,0.17)$ & 0.19 & $-0.38(-0.93,0.16)$ & 0.17 \\
\hline \multicolumn{5}{|l|}{ Volume measurement } \\
\hline Tricuspid regurgitation (Non-dialysis) & $-0.11(-0.76,0.54)$ & 0.74 & $-0.04(-0.70,0.62)$ & 0.90 \\
\hline Intradialytic weight gain (3-month average) & $5.84(-1.51,13.19)$ & 0.12 & $7.67(0.06,15.28)$ & 0.05 \\
\hline Intradialytic weight gain (Prior to study visit) & $2.03(-1.91,5.97)$ & 0.31 & $1.69(-2.33,5.71)$ & 0.41 \\
\hline
\end{tabular}

${ }^{a}$ Model 1 included non-dialysis seated systolic blood pressure, pulse wave velocity, central augmentation index, tricuspid regurgitation, intradialytic weight gain (3-month average), and intradialytic weight gain (prior to study visit), as well as baseline age, sex, ethnicity, body mass index, history of hypercholesterolemia, smoking, diabetes mellitus, coronary heart disease, congestive heart failure, cause of end-stage renal disease, time since first nephrology visit, beta blocker use, RAAS blockade use, number of antihypertensive medication, and dietary sodium intake

${ }^{\mathrm{b}}$ Model 2 included non-dialysis seated diastolic blood pressure, pulse wave velocity, central augmentation index, tricuspid regurgitation, intradialytic weight gain (3-month average), and intradialytic weight gain (prior to study visit), as well as baseline age, sex, ethnicity, body mass index, history of hypercholesterolemia, smoking, diabetes mellitus, coronary heart disease, congestive heart failure, cause of end-stage renal disease, time since first nephrology visit, beta blocker use, RAAS blockade use, number of antihypertensive medication, and dietary sodium intake

which accounts for a higher burden of arterial stiffness due to prolonged dialysis as well as the natural process of aging. Studies with prevalent patients also introduce a survivor bias, which needs to be considered when determining risk factors predicting cardiovascular disease. Though we did not observe a direct association between pulse wave velocity and LVMI, the association between higher pulse pressure, which is determined by arterial compliance and the intensity of wave reflections that are influenced by arterial stiffness, and higher LVMI suggests that reduced arterial compliance and distensibility may still have an underlying role and potentially contribute to LVH [40].

Lastly, IDWG is associated with LVMI, however, the association is attenuated after adjustment for other confounders. Another surrogate measure of volume status, pulmonary pressure, is not significantly associated with higher LVMI. In our study population of younger and diverse ethnicities, adjusting for dietary sodium intake does not modify any associations. The results of our study likely differ based on the change in residual kidney function of dialysis patients over years. Most of our cohort is studied within the first 6 months from diagnosis. This suggests that during the transition from reaching ESRD to receiving dialysis, IDWG management may not be a significant contributor until there is loss of residual function.

A few limitations of this study include the cross-sectional study design, which does not provide evidence of a temporal relationship and would require a trial to demonstrate normalization of blood pressure. Other measures of volume status and pulmonary pressure and preload should also be considered in future studies, as tricuspid regurgitation by echocardiography alone may not be as accurate as newer methods such as ultrasounds to detect lung congestion [41], and TR velocity does not clearly reflect LV preload in the presence of pulmonary vascular disease even though few had documented disease. Nonetheless, pulmonary hypertension is often observed together with volume overload, and has been associated with fluid volume status in past studies that showed a strong association between left atrial diameter and chronic volume overload [42]. Studies have also shown that after a series of ultrafiltration sessions, thereby treating volume overload, tricuspid regurgitation disappeared in most patients [43]. Furthermore, it is difficult to accurately measure volume status using one type of measurement; therefore we used additional factors such as IDWG, which is clinically relevant and available, to help estimate volume status.

Despite the limitations, this well characterized cohort of incident hemodialysis patients has been assessed in a consistent manner on non-dialysis days with standardized questions, adjudicated comorbidities and methods for cardiovascular assessment, as well as extensive dialysis unit BP and IDWG data. Moreover, our study cohort is predominantly African American (72.6 \%), often underrepresented in many studies or trials, despite 
comprising $10.4 \%$ of the Medicare end stage renal disease population. Understanding cardiovascular risk in this high risk population is important [44].

\section{Conclusion}

Among a younger and incident hemodialysis population, afterload as measured by BP using either predialysis or interdialytic measures, is most associated with higher LVMI. This suggests that future studies should consider the use of various $\mathrm{BP}$ measures in examining the impact of BP on LVM and cardiovascular disease. Findings from such studies could suggest that high BP should be more aggressively treated to promote LVH regression in incident hemodialysis patients.

\section{Additional file}

Additional file 1: Figure S1. Boxplot of left ventricular mass index (LVMI) and quartiles of systolic or diastolic blood pressures measured as predialysis 3-month average BP measurement stratified by ethnicity. Figure S2: Boxplot of left ventricular mass index (LVMI) and quartiles of systolic or diastolic blood pressures measured as prior to clinic BP measurement stratified by ethnicity. Figure S3: Boxplot of left ventricular mass index (LVMI) and quartiles of systolic or diastolic blood pressures measured as non-dialysis supine BP measurement stratified by ethnicity. Table S1: Independent associations of predialysis blood pressure, arterial, and volume measures with LVMI by linear regression among incident hemodialysis participants. Table S2: Independent associations of blood pressure prior to study visit, arterial, and volume measures withLVMI by linear regression among incident hemodialysis participants. Table S3: Independent associations of mean arterial pressure, arterial, and volume measures with LVMI by linear regression among incident hemodialysis participants. Table S4: Independent associations of pulse pressures, arterial, and volume measures with LVMI by linear regression among incident hemodialysis participants. Table S5: Association of preload and afterload measures with LVMI by linear regression among incident hemodialysis participants stratified by ethnicity. Table S6: Association of preload and afterload measures with LVMI by linear regression among incidenthemodialysis participants stratified by 3 month average IDWG groups. Table S7: Association of preload and afterload measures with LVMI by linear regression among incident hemodialysis participants stratified by $\beta$-blocker medication. Table S8: Association of preload and afterload measures with LVMI by linear regression among incident hemodialysis participants stratified by renin-angiotensin-aldosterone system (RAAS) blockade use. Table S9: Association of preload and afterload measures with LVMI by linear regression among incident hemodialysis participants stratified by history of congestive heart failure. Table S10: Association of hemoglobin with LVMI by linear regression among incident hemodialysis participants. (DOCX $97 \mathrm{~kb}$ )

\section{Abbreviations}

ACEi: Angiotensin converting enzyme inhibitor; ARB: Angiotensin-Il receptor blocker; BMI: Body-mass index; BP (SBP: DBP), Blood pressure (systolic blood pressure, diastolic blood pressure); CAD: Coronary artery disease; CCl: Charlson comorbidity index; CHF: Congestive heart failure; Cl: Confidence interval; CKD: Chronic kidney disease; ESRD: End-stage renal disease; HD: Hemodialysis; ICTR: Institute for Clinical and Translational Research; IDWG: Intradialytic weight gain; IVST: Interventricular septal thickness; LV: Left ventricle; LVH: Left ventricular hypertrophy; LVID: Left ventricular internal diameter; LVM: Left ventricular mass; LVMI: Left ventricular mass index; MAP: Mean arterial pressure; PACE study: Predictors of Arrhythmic and Cardiovascular Risk in End-Stage Renal Disease study: PVD: Peripheral vascular disease; PWT: Left ventricular wall thickness; PWV: Pulse wave velocity; RAAS: Renin-angiotensin-aldosterone system; SD: Standard deviation; TR: Tricuspid regurgitation; WHR: Waist to hip ratio.

\section{Competing interests}

The authors declare that they have no competing interest.

\section{Authors' contributions}

EK participated in study design, statistical analysis, data analysis and interpretation, and drafting of the manuscript. SS participated in analysis planning, data analysis and interpretation, and critical review of the manuscript. ME participated in analysis planning, data analysis sand interpretation, and critical review of the manuscript. BJ participated in analysis planning, data analysis and interpretation, and critical review of the manuscript. TS participated in analysis planning, data analysis and interpretation, and critical review of the manuscript. LM participated in analysis planning, data analysis and interpretation, and critical review of the manuscript. LK participated in study conception and design, and critical review of the manuscript. $J L$ participated in data analysis and interpretation, and critical review of the manuscript. RP participated in all stages of the project, including study conception and design, analysis planning, data analysis and interpretation, and critical review of the manuscript. All authors read and edited the manuscript for important content, and approved the final manuscript.

\section{Acknowledgements}

We dedicate this manuscript to the memory of our friend and colleague, Dr. W.H. Linda Kao, and her enduring work on improving patient outcomes in chronic disease.

We thank our participants, nephrologists and staff of the DaVita and MedStar dialysis units in the Baltimore area who contributed to the PACE study. We would like to thank the participation of dialysis practices in Baltimore, in particular, the Mid-Atlantic Nephrology Associates and the Nephrology Center of Maryland. We thank the PACE study and the Johns Hopkins GCRC staff for their effort, Kimberly Keck, and the members of the Data Safety Monitoring Board of the study, Drs. Paul Scheel, Luis Gimenez and Roger Blumenthal.

\section{Support}

The study and faculty were supported by the NIDDK-R01DK072367 (Parekh, Kao and Meoni), DK-083514 (Shafi), and the National Center for Research Resources - NIH Roadmap for Medical Research.

\section{Author details}

'Division of Epidemiology, Dalla Lana School of Public Health, University of Toronto, Toronto, Ontario, Canada. ${ }^{2}$ Department of Medicine, School of Medicine, Johns Hopkins University, Baltimore, Maryland, USA. ${ }^{3}$ Welch Center for Prevention, Epidemiology and Clinical Research, Johns Hopkins University, Baltimore, Maryland, USA. ${ }^{4}$ Nephrology Center of Maryland, Baltimore, Maryland, USA. ${ }^{5}$ Department of Biostatistics, Bloomberg School of Public Health, Johns Hopkins University, Baltimore, Maryland, USA. 'Department of Epidemiology, Bloomberg School of Public Health, Johns Hopkins University, Baltimore, Maryland, USA. ${ }^{7}$ Department of Pediatrics and Medicine, School of Medicine, University of Toronto, Toronto, Ontario, Canada. ${ }^{8}$ Hospital for Sick Children, University Health Network and University of Toronto, Toronto, ON, Canada.

Received: 24 December 2014 Accepted: 28 July 2015 Published online: 07 August 2015

\section{References}

1. Foley RN, Herzog CA, Collins AJ, United States Renal Data S. Blood pressure and long-term mortality in United States hemodialysis patients: USRDS Waves 3 and 4 Study. Kidney Int. 2002;62(5):1784-90.

2. London GM, Guerin AP, Marchais SJ, Pannier B, Safar ME, Day M, et al. Cardiac and arterial interactions in end-stage renal disease. Kidney Int. 1996;50(2):600-8.

3. Matsumoto M, lo H, Furukawa M, Okumura K, Masuda A, Seto T, et al. Risk factors associated with increased left ventricular mass index in chronic kidney disease patients evaluated using echocardiography. J Nephrol. 2012;25(5):794-801.

4. Harvey PR, Holt A, Nicholas J, Dasgupta I. Is an average of routine postdialysis blood pressure a good indicator of blood pressure control and cardiovascular risk? J Nephrol. 2013;26(1):94-100. 
5. Foley RN, Curtis BM, Randell EW, Parfrey PS. Left ventricular hypertrophy in new hemodialysis patients without symptomatic cardiac disease. Clin J Am Soc Nephrol. 2010;5(5):805-13.

6. Li Z, Lacson Jr E, Lowrie EG, Ofsthun NJ, Kuhlmann MK, Lazarus JM, et al. The epidemiology of systolic blood pressure and death risk in hemodialysis patients. Am J Kidney Dis. 2006;48(4):606-15.

7. Agarwal R, Lewis RR. Prediction of hypertension in chronic hemodialysis patients. Kidney Int. 2001;60(5):1982-9.

8. Verdecchia P. Prognostic value of ambulatory blood pressure : current evidence and clinical implications. Hypertension. 2000;35(3):844-51.

9. Blacher J, Guerin AP, Pannier B, Marchais SJ, Safar ME, London GM. Impact of aortic stiffness on survival in end-stage renal disease. Circulation. 1999:99(18):2434-9.

10. Laurent S, Boutouyrie P, Asmar R, Gautier I, Laloux B, Guize L, et al. Aortic stiffness is an independent predictor of all-cause and cardiovascular mortality in hypertensive patients. Hypertension. 2001;37(5):1236-41

11. Guerin AP, Blacher J, Pannier B, Marchais SJ, Safar ME, London GM. Impact of aortic stiffness attenuation on survival of patients in end-stage renal failure. Circulation. 2001;103(7):987-92

12. London GM, Marchais SJ, Guerin AP. Arterial stiffness and function in end-stage renal disease. Adv Chronic Kid Dis. 2004;11(2):202-9.

13. Vertes V, Cangiano JL, Berman LB, Gould A. Hypertension in end-stage renal disease. N Engl J Med. 1969;280(18):978-81.

14. Hur E, Usta M, Toz H, Asci G, Wabel P, Kahvecioglu S, et al. Effect of fluid management guided by bioimpedance spectroscopy on cardiovascular parameters in hemodialysis patients: a randomized controlled trial. Am J Kidney Dis. 2013;61(6):957-65.

15. Nitta K, Akiba T, Uchida K, Otsubo S, Otsubo Y, Takei T, et al. Left ventricular hypertrophy is associated with arterial stiffness and vascular calcification in hemodialysis patients. Hypertens Res. 2004;27(1):47-52.

16. Parekh RS, Meoni LA, Jaar BG, Sozio SM, Shafi T, Tomaselli GF, et al. Rationale and design for the Predictors of Arrhythmic and Cardiovascular Risk in End Stage Renal Disease (PACE) study. BMC Nephrol. 2015;16:63.

17. Armstrong AC, Gidding S, Gjesdal O, Wu C, Bluemke DA, Lima JA. LV mass assessed by echocardiography and CMR, cardiovascular outcomes, and medical practice. J Am Coll Cardiol Img. 2012;5(8):837-48.

18. Townsend RR, Wimmer NJ, Chirinos JA, Parsa A, Weir M, Perumal K, et al. Aortic PWV in chronic kidney disease: a CRIC ancillary study. Am J Hypertens. 2010;23(3):282-9.

19. Lanzarini L, Fontana A, Lucca E, Campana C, Klersy C. Noninvasive estimation of both systolic and diastolic pulmonary artery pressure from Doppler analysis of tricuspid regurgitant velocity spectrum in patients with chronic heart failure. Am Heart J. 2002;144(6):1087-94.

20. Mutlak D, Aronson D, Lessick J, Reisner SA, Dabbah S, Agmon Y. Functional tricuspid regurgitation in patients with pulmonary hypertension: is pulmonary artery pressure the only determinant of regurgitation severity? Chest. 2009;135(1):115-21.

21. Stephen B, Dalal P, Berger M, Schweitzer P, Hecht S. Noninvasive estimation of pulmonary artery diastolic pressure in patients with tricuspid regurgitation by Doppler echocardiography. Chest. 1999;116(1):73-7.

22. Bolignano D, Rastelli S, Agarwal R, Fliser D, Massy Z, Ortiz A, et al. Pulmonary hypertension in CKD. Am J Kidney Dis. 2013:61(4):612-22.

23. Agarwal R, Bouldin JM, Light RP, Garg A. Inferior vena cava diameter and left atrial diameter measure volume but not dry weight. Clin J Am Soc Nephrol. 2011;6(5):1066-72

24. Devereux RB, Alonso DR, Lutas EM, Gottlieb GJ, Campo E, Sachs I, et al. Echocardiographic assessment of left ventricular hypertrophy: comparison to necropsy findings. Am J Cardiol. 1986;57(6):450-8.

25. Royston $P$, White IR. Multiple Imputation by Chained Equations (MICE): Implementation in Stata. J Stat Softw. 2011;45(4):1-20.

26. Agarwal R, Sinha AD, Pappas MK, Abraham TN, Tegegne GG. Hypertension in hemodialysis patients treated with atenolol or lisinopril: a randomized controlled trial. Nephrol Dialy Trans. 2014;29(3):672-81.

27. Bansal N, Keane M, Delafontaine P, Dries D, Foster E, Gadegbeku CA, et al. A longitudinal study of left ventricular function and structure from CKD to ESRD: the CRIC study. Clin J Am Soc Nephrol. 2013;8(3):355-62.
28. Agarwal R. Managing hypertension using home blood pressure monitoring among haemodialysis patients-a call to action. Nephrol Dial Trans. 2010;25(6):1766-71

29. Agarwal R, Flynn J, Pogue V, Rahman M, Reisin E, Weir MR. Assessment and management of hypertension in patients on dialysis. J Am Soc Nephrol JASN. 2014:25:1630-46.

30. Levin NW, Kotanko P, Eckardt KU, Kasiske BL, Chazot C, Cheung AK, et al. Blood pressure in chronic kidney disease stage 5D-report from a kidney disease: improving global outcomes controversies conference. Kidney Int. 2010;77(4):273-84

31. Agarwal R, Brim NJ, Mahenthiran J, Andersen MJ, Saha C. Out-of-hemodialysis -unit blood pressure is a superior determinant of left ventricular hypertrophy. Hypertension. 2006:47(1):62-8.

32. Conion PJ, Walshe JJ, Heinle SK, Minda S, Krucoff M, Schwab SJ. Predialysis systolic blood pressure correlates strongly with mean 24-hour systolic blood pressure and left ventricular mass in stable hemodialysis patients. J Am Soc Nephrol. 1996;7(12):2658-63.

33. Klassen PS, Lowrie EG, Reddan DN, DeLong ER, Coladonato JA, Szczech LA et al. Association between pulse pressure and mortality in patients undergoing maintenance hemodialysis. JAMA. 2002;287(12):1548-55.

34. Zhang K, Chen J, Liu Y, Wang T, Wang L, Wang J, et al. Diastolic blood pressure reduction contributes more to the regression of left ventricular hypertrophy: a meta-analysis of randomized controlled trials. J Hum Hypertens. 2013;27(11):698-706.

35. Workgroup KD. K/DOQI clinical practice guidelines for cardiovascular disease in dialysis patients. Am J Kidney Dis. 2005:45(4 Suppl 3):S1-153.

36. James PA, Oparil S, Carter BL, Cushman WC, Dennison-Himmelfarb C, Handler J, et al. 2014 evidence-based guideline for the management of high blood pressure in adults: report from the panel members appointed to the Eighth Joint National Committee (JNC 8). JAMA. 2014;311(5):507-20.

37. Cannella G, Paoletti E, Delfino R, Peloso G, Molinari S, Traverso GB. Regression of left ventricular hypertrophy in hypertensive dialyzed uremic patients on long-term antihypertensive therapy. Kidney Int. 1993;44(4):881-6.

38. Tai DJ, Lim TW, James MT, Manns BJ, Tonelli M, Hemmelgarn BR, et al. Cardiovascular effects of angiotensin converting enzyme inhibition or angiotensin receptor blockade in hemodialysis: a meta-analysis. Clin J Am Soc Nephrol. 2010;5(4):623-30.

39. Blacher J, Guerin AP, Pannier B, Marchais SJ, London GM. Arteria calcifications, arterial stiffness, and cardiovascular risk in end-stage renal disease. Hypertension. 2001;38(4):938-42.

40. Muller-Brunotte R, Kahan T, Malmqvist K, Edner M. Swedish ibesartan left ventricular hypertrophy investigation vs a: Blood pressure and left ventricular geometric pattern determine diastolic function in hypertensive myocardial hypertrophy. J Hum Hypertens. 2003;17(12):841-9.

41. Zoccali C, Torino C, Tripepi R, Tripepi G, D'Arrigo G, Postorino M, et al. Pulmonary congestion predicts cardiac events and mortality in ESRD. J Am Soc Nephrol. 2013;24(4):639-46.

42. Kim SC, Chang HJ, Kim MG, Jo SK, Cho WY, Kim HK. Relationship between pulmonary hypertension, peripheral vascular calcification, and major cardiovascular events in dialysis patients. Kidney Res Clin Pract. 2015;34(1):28-34

43. Cirit M, Ozkahya M, Cinar CS, Ok E, Aydin S, Akcicek F, et al. Disappearance of mitral and tricuspid regurgitation in haemodialysis patients after ultrafiltration. Nephrol Dial Trans. 1998:13(2):389-92.

44. Lloyd-Jones D, Adams RJ, Brown TM, Carnethon M, Dai S, De Simone G, et al. Executive summary: heart disease and stroke statistics-2010 update: a report from the American Heart Association. Circulation. 2010;121(7):948-54. 